\title{
Effects of Sitagliptin on Pancreatic $\beta$ Cell Function and Microangiopathy in Japanese Patients With Type 2 Diabetes Mellitus: Follow-Up for 4 Years
}

\author{
Hiromi Hamamoto ${ }^{\mathrm{a}, \mathrm{d}}$, Hiroyuki Okada ${ }^{\mathrm{b}}$, Mitsuhiko Noda ${ }^{\mathrm{c}}$
}

\begin{abstract}
Background: This study was aimed at investigating the effect of longterm sitagliptin treatment in improving the pancreatic $\beta$ cell function and its influence on microangiopathy in patients with type 2 diabetes mellitus.

Methods: The study was designed as a retrospective analysis of the data of 27 patients with type 2 diabetes mellitus who did not have any evident reduction of the renal function and had received sitagliptin treatment for 4 years or longer. The fasting plasma C-peptide level corrected for the fasting blood glucose level (C-peptide index (CPI)), hemoglobin A1c (HbAlc) and body weight were determined every year during the 4-year period, and the status of retinopathy and nephropathy at the end of the fourth year of sitagliptin treatment was compared with the pre-treatment status.
\end{abstract}

Results: Both the HbA1c and body weight were significantly decreased by 6 months after the start of treatment. Thereafter, the $\mathrm{HbA} 1 \mathrm{c}$ showed no further rise during the subsequent 4-year period, while the body weight continued to decrease over the 4-year period. No significant change of the CPI, as compared to the pre-treatment level $(0.95 \pm 0.49)$, was observed at any time during the follow-up. The retinopathy and nephropathy remained unchanged in severity in most cases; however, progression of retinopathy was seen in seven cases $(29 \%)$ and that of nephropathy in three cases (11\%).

Conclusions: Maintenance of good blood glucose control for 4 years by sitagliptin treatment allowed the pancreatic $\beta$ cell function to be preserved. However, it was not possible to suppress progression of microangiopathy completely by treatment for a short period of 4 years.

Manuscript accepted for publication August 14, 2015

${ }^{a}$ Department of Internal Medicine, Fukuyama City Hospital, Hiroshima, Japan ${ }^{b}$ Department of Gastroenterology and Hepatology, Okayama University Graduate School of Medicine, Dentistry, and Pharmaceutical Sciences, Okayama, Japan

'Department of Diabetes Research Center, National Center for Global Health and Medicine, Tokyo, Japan

${ }^{\mathrm{d} C o r r e s p o n d i n g ~ A u t h o r: ~ H i r o m i ~ H a m a m o t o, ~ D e p a r t m e n t ~ o f ~ I n t e r n a l ~ M e d i c i n e, ~}$ Fukuyama City Hospital, 5-23-1 Zao, Fukuyama, Hiroshima 721-8511, Japan. Email: hihamamoto@city.fukuyama.hiroshima.jp

doi: http://dx.doi.org/10.14740/jem297w
Keywords: Sitagliptin; Pancreatic $\beta$ cell function; Microangiopathy; Japanese patients; Type 2 diabetes mellitus

\section{Introduction}

In the treatment of type 2 diabetes mellitus, attempt to maintain the pancreatic $\beta$ cell function is an important issue. According to the report of the UKPDS16 [1], pancreatic $\beta$ cell function improves slightly 1 year after the start of treatment of type 2 diabetes mellitus with any of insulin, a sulfonylurea (SU) or a biguanide; however, the function begins to decrease from the second year onward, showing a $4 \%$ reduction annually. Similar results have also been reported in Japanese patients by Funakoshi et al [2]. After the clinical introduction of dipeptidyl peptidase-4 (DPP-4) inhibitors, Seck et al [3] reported preservation of the pancreatic $\beta$ cell function until 2 years after the start of sitagliptin treatment, but there are no reports on longer-term outcomes or the outcomes in Japanese patients, who are known to have lower insulin-secreting potential than Western patients. Under such circumstances, we recently conducted a retrospective evaluation of changes in the pancreatic $\beta$ cell function following sitagliptin treatment for a 4-year period through analysis of the C-peptide index (CPI), i.e. fasting serum C-peptide level corrected for the fasting blood glucose level.

\section{Patients and Methods}

Initially, using our department diabetes database, we conducted a chart review of type 2 diabetes mellitus patients who had received sitagliptin (MSD K.K. a subsidiary of Merck \& Co., Inc., Kenilworth, NJ, USA) treatment $(50 \mathrm{mg} /$ day) at our hospital for 4 years or longer with oral informed consent. The dosage was $50 \mathrm{mg}$ once in the morning, which is the recommended dose for patients without renal impairment in Japan. Of the 119 patients who began to receive sitagliptin treatment between January 2010 and April 2011, the 86 patients who discontinued receiving treatment at our facility within 4 years after the start (for reasons of referral to other facilities (50), no effect (13), withdrawal (nine), drop-out (six), cancer (four), or death (four)) were excluded, and the data of the remaining 33 patients were extracted for this study. Of these 33 patients, four 
Table 1. Baseline Characteristics

\begin{tabular}{|c|c|}
\hline \multicolumn{2}{|l|}{ Characteristic } \\
\hline Patients, $\mathrm{n}$ & 27 \\
\hline Age, years (mean $\pm \mathrm{SD})$ & $63.3 \pm 9.2$ \\
\hline Male, n (\%) & $20(74)$ \\
\hline \multicolumn{2}{|l|}{ Duration of diabetes, n (\%) } \\
\hline$<5$ years & $6(16)$ \\
\hline $5-9$ years & $9(33)$ \\
\hline $10-19$ years & $7(26)$ \\
\hline$\geq 20$ years & $3(11)$ \\
\hline Unknown & $2(7)$ \\
\hline $\mathrm{BMI}, \mathrm{kg} / \mathrm{m}^{2}($ mean $\pm \mathrm{SD})$ & $22.9 \pm 3.0$ \\
\hline HbAlc (\%) (mean \pm SD) & $7.2 \pm 1.8$ \\
\hline $\mathrm{CPI}($ mean $\pm \mathrm{SD})$ & $0.95 \pm 0.49$ \\
\hline \multicolumn{2}{|l|}{ Diabetic retinopathy, n (\%) } \\
\hline NDR & $15(56)$ \\
\hline SDR & $8(30)$ \\
\hline PPDR & $2(7)$ \\
\hline PDR & $0(0)$ \\
\hline Unknown & $2(7)$ \\
\hline \multicolumn{2}{|l|}{ Diabetic nephropathy, n (\%) } \\
\hline Stage 1 & $19(70)$ \\
\hline Stage 2 & $7(26)$ \\
\hline Stage 3 & $1(4)$ \\
\hline Stage 4 & $0(0)$ \\
\hline \multicolumn{2}{|l|}{ Diabetes treatment, n (\%) } \\
\hline None & $19(70)$ \\
\hline Insulin & $3(11)$ \\
\hline Sulfonylureas & $9(33)$ \\
\hline Glinides & $13(48)$ \\
\hline Biguanides & $7(26)$ \\
\hline Thiazolidinediones & $8(30)$ \\
\hline$\alpha$-Glucosidase inhibitors & $14(52)$ \\
\hline
\end{tabular}

Data are means \pm SD or $n(\%)$. NDR: no diabetic retinopathy; SDR: simple diabetic retinopathy; PPDR: pre-proliferative diabetic retinopathy; PDR: proliferative diabetic retinopathy. Stage 1: normoalbuminuria. Stage 2: microalbuminuria. Stage 3: macroalbuminuria. Stage 4: renal insufficiency.

patients who had compromised renal function (creatinine $(\mathrm{Cr})$ $\geq 1.1$ or estimated glomerular filtration rate $(\mathrm{eGFR})<50)$ or two patients who had not undergone $\mathrm{C}$-peptide measurement at the start of the treatment were excluded, and the data of the remaining 27 patients were included in this analysis. Analysis also included eight patients in whom insulin secretion stimulators were discontinued or continued at reduced dose levels after the start of sitagliptin treatment and patients in whom improvement/worsening of hemoglobin A1c (HbA1c) during the course of sitagliptin treatment required dose change, addition or discontinuation of hypoglycemic drugs other than sitagliptin. This study was not in conflict with principles expressed in the Declaration of Helsinki.

In these patients, investigation based on the medical records was conducted to determine the fasting C-peptide level, $\mathrm{HbA} 1 \mathrm{c}$ and body weight at the start of treatment, 6 months after the start of treatment, and every 12 months thereafter until the end of 4 years of treatment. The blood samples were collected after overnight fasting. HbA1c levels were measured using a Latex agglutination reaction method kit (Kyowa Medex Co., Ltd, Tokyo, Japan) with a normal range of 4.6-6.2\%. C-peptide levels were measured using a chemiluminescent immunoassay (CLEIA) kit (SIEMENS Healthcare Diagnostics, Tokyo, Japan) with a normal range of $0.74-3.48 \mathrm{ng} / \mathrm{mL}$. The fasting C-peptide level was corrected for the fasting blood glucose level to obtain the CPI $(\mathrm{CPI}=$ fasting serum C-peptide $(\mathrm{ng} / \mathrm{mL})$ $\times 100$ /fasting plasma glucose $(\mathrm{mg} / \mathrm{dL}))$. In regard to the data for the months in which the patient did not visit or in which the measurements were not conducted, we replenished the C-peptide data using the data collected within 3 months before or after the month concerned, and replenished the body mass index (BMI) and HbAlc data with the mean for the two neighboring months (one month before and after the data-lacking month).

Changes in the stage of microangiopathy were analyzed by determining the changes in the severity of retinopathy and nephropathy between the start of treatment and the end of 4 years of treatment. The severity of retinopathy was rated on a four-grade scale based on the Davis classification: no diabetic retinopathy (NDR), simple diabetic retinopathy (SDR), pre-proliferative diabetic retinopathy (PPDR) and proliferative diabetic retinopathy (PDR). The severity of nephropathy was rated on a five-stage scale (stage 1 to 5) in accordance with the classification of Diabetic Nephropathy of the Joint Committee on Diabetic Nephropathy.

The data on each parameter after the start of treatment were compared to the pre-treatment data by a paired $t$-test. $\mathrm{P}<$ 0.05 was regarded as statistically significant.

\section{Results}

Table 1 summarizes the background variables at the baseline (age, gender, body weight, duration of illness, microangiopathy, treatment received, and laboratory data).

No significant change of the CPI (mean \pm SD) from the pre-treatment level was observed during the treatment for 4 years $(0.88 \pm 0.28$ at 6 months, $0.90 \pm 0.38$ at 1 year, $0.89 \pm 0.62$ at 2 years, $0.93 \pm 0.41$ at 3 years, and $0.88 \pm 0.33$ at 4 years) (Fig. 1A). The HbA1c decreased significantly to $6.3 \pm 0.5 \%$ at 6 months $(\mathrm{P}=0.03)$, remaining low thereafter until 4 years $(6.4 \pm 0.5 \%$ at 1 year $(\mathrm{P}=0.03), 6.4 \pm 0.5 \%$ at 2 years $(\mathrm{P}=0.04)$, $6.3 \pm 0.4 \%$ at 3 years $(\mathrm{P}=0.02)$, and $6.3 \pm 0.4 \%$ at 4 years $(\mathrm{P}$ $=0.03)$ ) (Fig. 1B). The body weight also showed significant decrease at 6 months to $-1.1 \pm 2.5 \mathrm{~kg}(\mathrm{P}=0.03)$ and continued to decrease thereafter until 4 years $(-1.4 \pm 2.9 \mathrm{~kg}$ at 1 year $(\mathrm{P}=$ $0.02),-1.6 \pm 3.1 \mathrm{~kg}$ at 2 years $(\mathrm{P}=0.01),-1.9 \pm 3.5 \mathrm{~kg}$ at 3 years $(\mathrm{P}=0.01)$, and $-2.8 \pm 4.3 \mathrm{~kg}$ at 4 years $(\mathrm{P}=0.002))($ Fig. $1 \mathrm{C})$. 

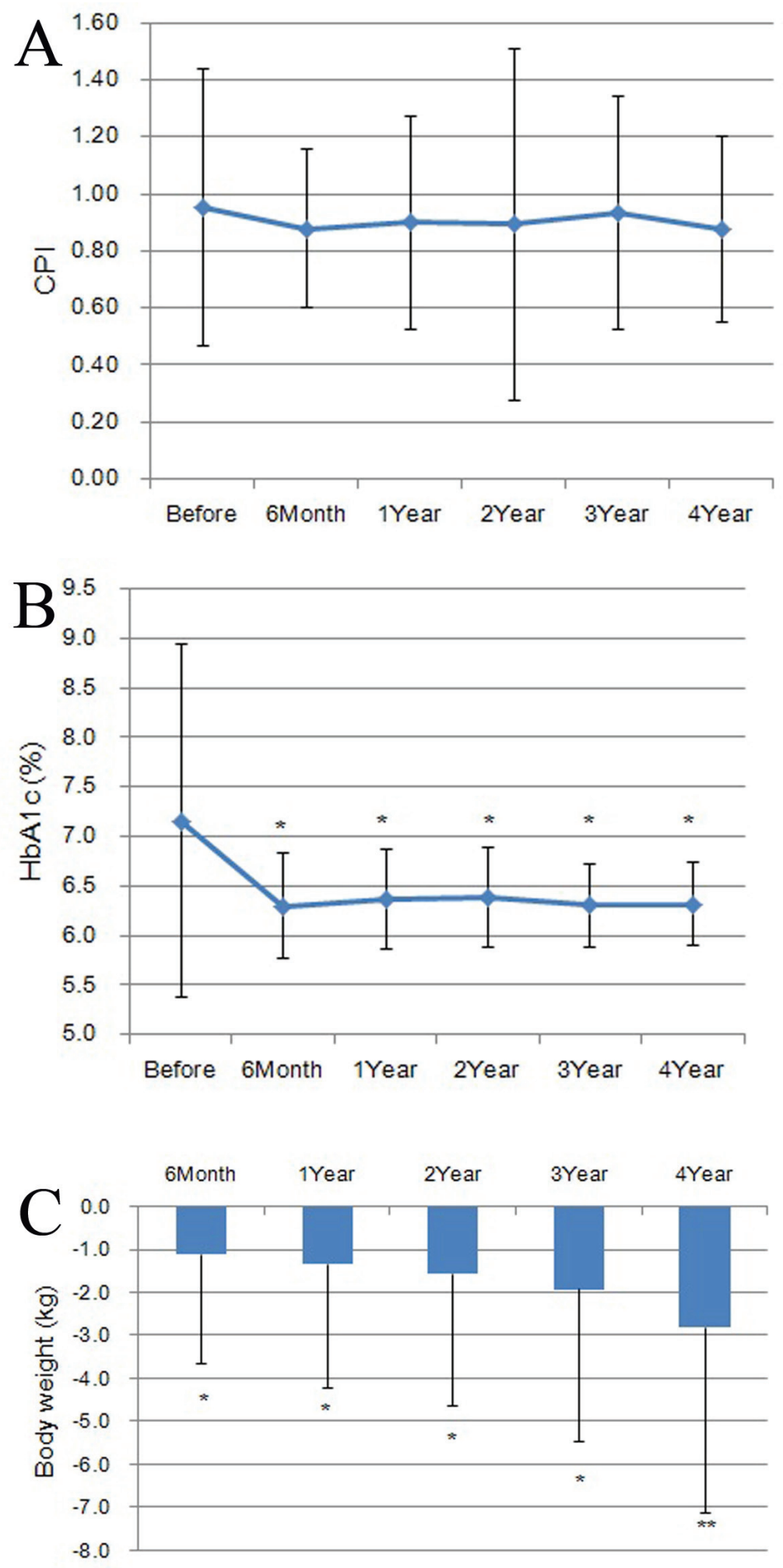

Figure 1. Changes in $\mathrm{CPI}(\mathrm{A}), \mathrm{HbA} 1 \mathrm{c}(\mathrm{B})$, and body weight $(\mathrm{C})$ during the treatment for 4 years. $(\mathrm{A}) \mathrm{CPI}$ showed no significant change from the pre-treatment level at any point of time after the start of sitagliptin treatment. (B) HbA1c decreased significantly at 6 months and remained reduced thereafter until 4 years. (C) Body weight decreased significantly at 6 months and continued to decrease thereafter until 4 years. 
Table 2. Changes in Retinopathy and Nephropathy After the 4-Year Treatment Period

\begin{tabular}{ll}
\hline & $\mathbf{n}(\mathbf{\%})$ \\
A. Retinopathy $(\mathrm{n}=24)$ & \\
Improve & $1(4)$ \\
No change & $16(67)$ \\
Worse & $7(29)$ \\
B. Nephropathy $(\mathrm{n}=27)$ & \\
Improve & $4(15)$ \\
No change & $20(74)$ \\
Worse & $3(11)$ \\
\hline
\end{tabular}

Data are $\mathrm{n}(\%)$. Frequency of outcomes of retinopathy: no change $>$ worse $>$ improve (A). Frequency of outcomes of nephropathy: no change $>$ improve $>$ worse $(B)$.

In the analysis of the changes in the stage of microangiopathy, the stage of nephropathy improved in four patients $(14.8 \%)$, remained unchanged in 20 patients $(74.1 \%)$, and worsened in three patients (11.1\%) (Table 2A). Changes in the stage of retinopathy were analyzed in 24 patients, after excluding three patients without sufficient data, revealing improvement in one patient $(4.2 \%)$, no change in 16 patients $(66.7 \%)$ and progression in seven patients $(29.2 \%)$. Thus, retinopathy advanced more frequently than nephropathy (Table 2B).

\section{Discussion}

Sitagliptin has been shown in rats to suppress the expression of mediator proteins involved in the apoptotic machinery and inflammation, and to stimulate the expression of mediator proteins involved in angiogenesis/proliferation [4]. This drug is expected to also increase the pancreatic $\beta$ cell population in humans. Based on these previous reports, we anticipated at the start of this study that the $\beta$ cell function would improve year after year following the start of sitagliptin treatment. In practice, however, the function remained unchanged, although it did not decrease, resembling the results of the 2-year study reported by Seck et al [3]. This result is probably attributable to the relatively short treatment period, namely, the 4-year treatment was probably insufficient for the pancreatic $\beta$ cell function-improving effect of this drug to be manifested in humans, although the effect in rats was observed just 6 weeks after the start of treatment. Notwithstanding this result, the pancreatic $\beta$ cell function was maintained, without showing deterioration, during the 4-year treatment period. This suggests the possibility that the drug suppressed collapse of the $\beta$ cell function indirectly through inducing good control of the blood glucose for a long term. These results from this study may be valuable in view of the previous report that good blood glucose control can be expected just by preservation of the pancreatic $\beta$ cell function, even without improvement of the function [5].

In regard to microangiopathy, the disease stage advanced in some cases despite preservation of the pancreatic $\beta$ cell function and good control of the HbAlc. Considering the re- port of Lachin et al [6] that while the fasting CPI was not correlated with the outcomes of diabetic complications, the postprandial or post-glucagon load C-peptide level was correlated with the risk of occurrence of complications, it seems likely that the post-prandial CPI decreased over time in our patients who showed progression of microangiopathy. If post-prandial CPI decrease is absent, the progression of microangiopathy may be attributable to an insufficient length of the survey period in the present study as compared to past studies (7 years for the evaluation of complications in the diabetes control and complications trial (DCCT)/the epidemiology of diabetes interventions and complications (EDIC) [6]; 6 years [1] plus an additional 10 years [7] in the UKPDS) or attributable to the negative legacy effect of the previous poor control period [7].

The present study, which was designed as a retrospective survey, has limitations. Firstly, we did not prepare appropriate control patients since we extracted only patients who had received sitagliptin. It is unclear whether differences exist or not between patients with sitagliptin and others. Secondly, many patients dropped out of the study because of good control of blood glucose levels, resulting in referral to other facilities. If these patients had been followed up until the end of the study, different results might have been obtained. Thirdly, this study includes eight patients who had some changes in insulin secretagogue other than sitagliptin. Therefore, C-peptide values might have been influenced when insulin secretagogue was added or discontinued or the dose was increased or decreased.

In conclusion, sitagliptin for a period of 4 years yielded good blood glucose control and allowed the pancreatic $\beta$ cell function to be preserved, although progression of microangiopathy was not completely suppressed. Thus, sitagliptin is promising as a drug for the treatment of type 2 diabetes mellitus.

\section{Acknowledgement}

The authors would like to thank Dr. Koji Nakanishi, for his advice. Mitsuhiko Noda has received speaker honoraria from Sanofi, Mitsubishi Tanabe Pharma, Daiichi Sankyo, Eli Lilly Japan, MSD, Sanwa Kagaku Kenkyusho, Ono Pharmaceutical Co. Ltd, Takeda Pharmaceutical Co. Ltd, Astellas, Kowa Pharmaceutical Co. Ltd, Taisho Toyama Pharmaceutical Co. Ltd, Kissei Pharmaceutical Co. Ltd, Meiji Seika Pharma Co. Ltd, Kyowa Hakko Kirin Co. Ltd, ABBVie Inc. and Johnson \& Johnson K.K., and research grants from Takeda Pharmaceutical Co. Ltd, Daiichi Sankyo, Mitsubishi Tanabe Pharma and Kyowa Hakko Kirin Co. Ltd.

\section{Grant Support}

None.

\section{References}

1. U.K. prospective diabetes study 16 . Overview of 6 years' therapy of type II diabetes: a progressive dis- 
ease. U.K. Prospective Diabetes Study Group. Diabetes. 1995;44(11):1249-1258.

2. Funakoshi S, Fujimoto S, Hamasaki A, Fujiwara H, Fujita Y, Ikeda K, Hamamoto Y, et al. Analysis of factors influencing pancreatic $\beta$-cell function in Japanese patients with type 2 diabetes: association with body mass index and duration of diabetic exposure. Diabetes Res Clin Pract. 2008;82(3):353-358.

3. Seck T, Nauck M, Sheng D, Sunga S, Davies MJ, Stein PP, Kaufman KD, et al. Safety and efficacy of treatment with sitagliptin or glipizide in patients with type 2 diabetes inadequately controlled on metformin: a 2-year study. Int J Clin Pract. 2010;64(5):562-576.

4. Mega C, Vala H, Rodrigues-Santos P, Oliveira J, Teixeira F, Fernandes R, Reis F, et al. Sitagliptin prevents ag- gravation of endocrine and exocrine pancreatic damage in the Zucker Diabetic Fatty rat - focus on amelioration of metabolic profile and tissue cytoprotective properties. Diabetol Metab Syndr. 2014;6(1):42.

5. Saisho Y, Kou K, Tanaka K, Abe T, Shimada A, Kawai T, Itoh $\mathrm{H}$. Association between beta cell function and future glycemic control in patients with type 2 diabetes. Endocr J. 2013;60(4):517-523.

6. Lachin JM, McGee P, Palmer JP. Impact of C-peptide preservation on metabolic and clinical outcomes in the Diabetes Control and Complications Trial. Diabetes. 2014;63(2):739-748.

7. Holman RR, Paul SK, Bethel MA, Matthews DR, Neil HA. 10-year follow-up of intensive glucose control in type 2 diabetes. N Engl J Med. 2008;359(15):1577-1589. 